\title{
TIPE2 acts as a biomarker for GIST risk category and suppresses the viability and invasiveness of GIST cells
}

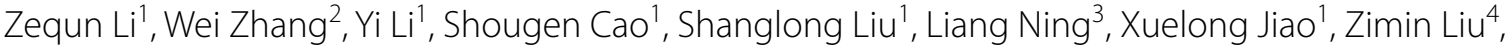 \\ Xiaoming Xing ${ }^{5}$, Yujun $\mathrm{Li}^{5}$ and Yanbing Zhou ${ }^{1 *}$
}

\begin{abstract}
Evaluating the risk category of gastrointestinal stromal tumors (GISTs) is crucial for predicting prognosis and choosing treatment strategies, and tumor metastasis usually represent poor prognosis. Tumor necrosis factor-alpha-induced protein 8-like 2 (TIPE2) is a novel described tumor suppressor. In the present study, TIPE2 expression was detected using a total of 96 human GIST specimens by immunohistochemistry. The effect of TIPE2 on proliferation and invasiveness of GIST cells and its related mechanisms were explored in vitro. It was found that TIPE2 expression was gradually decreased in accordance with GIST risk grades and negatively associated with tumor size, mitotic count and risk category. Moreover, TIPE2 was identified as a biomarker for evaluating the risk grade of GIST. TIPE2 markedly suppressed the viability, colony formation, migration and invasion of GIST cells. Furthermore, TIPE2 induced apoptosis and suppressed MMP-9 expression of GIST cells by targeting Rac1. In conclusion, these results indicate that TIPE2 plays a pivotal role in the progression of GIST. TIPE2 serves as a promising biomarker for evaluating GIST risk grade and a potential target for treatment of GIST.
\end{abstract}

Keywords: TIPE2, GIST, Risk category, Rac1

\section{Introduction}

GISTs are mesenchymal neoplasms that arise in the gastrointestinal (GI) tract [1-3]. According to the modified NIH criteria, the distribution of risk for primary GIST is categorized as very low risk (15\%), low risk (30\%), intermediate risk (22\%) and high risk (33\%), which is determined by tumor mitotic rate and size, tumor site, and presence of tumor rupture [4]. Till now, surgery remains mainstay of treatment for GIST patients. More than half of the GIST patients ultimately develop local recurrence or distant metastasis, which often leads to fatal prognosis [1, 5-7]. Currently, the criteria for risk evaluation depend largely on clinicopathologic factors such as tumor size and mitotic rate, which cannot predict the risk

\footnotetext{
*Correspondence: zhouyanbing999@aliyun.com

1 Department of General Surgery, Affiliated Hospital of Qingdao University, 16\# Jiangsu Road, Qingdao, Shandong, People's Republic of China

Full list of author information is available at the end of the article
}

of malignancy accurately $[2,8]$. The detailed molecular mechanisms of this mesenchymal neoplasm are poorly understood. Therefore, discovering novel biomarkers and a better understanding of the detailed molecular mechanisms for the progression of GIST is urgently needed.

Tumor necrosis factor (TNF)- $\alpha$-induced protein 8 (TNFAIP8) like 2 belongs to the TNFAIP8 family [9]. TIPE2 was initially identified as a negative immune regulator that is of vital importance in maintaining immune homeostasis [10, 11]. It has been demonstrated that TIPE2 is down-regulated in patients with infectious or autoimmune diseases [12-14]. Moreover, recent research found that TIPE2 was also involved in the development of a variety of tumors [15-18]. Our previous research found that by inhibiting the activity of Rac1, TIPE2 could suppress the tumorigenesis and development of tumor cells in HCC and NSCLC [15, 17-19].

Rac1 belongs to the Ras superfamily of small GTPases, research found that Rac1 overexpression is correlated with poor prognosis in a number of tumors [20,21]. Rac1 
plays a pivotal role in tumor development, especially in the invasiveness and metastasis of tumors [22, 23]. Several classical molecules associated with tumor invasiveness, such as MMPs and uPA, have been demonstrated to be the downstream effectors of Rac1 signaling [24]. Moreover, it has also been demonstrated that Rac1 is involved in regulating apoptosis of tumor cells $[25,26]$.

In the present study, we detected the expression of TIPE2 in GIST tissues and its clinical significance in evaluating GIST risk category. Moreover, in vivo experiments were conducted to explore the effect of TIPE2 on the malignant behaviors of GIST cells. Furthermore, we also investigated the related mechanisms for the aforementioned effect of TIPE2.

\section{Materials and methods}

\section{Patients and tissues samples}

A total of 96 human GIST specimens were collected from Department of General Surgery, Affiliated Hospital of Qingdao University. Immunohistochemical staining for CD117, CD34, DOG1, Ki-67 were conducted by the Department of Pathology to confirm the diagnosis of GIST and evaluate the risk category. According to the modified NIH criteria, the number of very low risk, low risk, intermediate risk and high risk were 22, 35, 18 and 21, respectively. All the experimental protocols were approved by the Ethics Committee of Qingdao University, China. Written informed consent was obtained from all subjects.

\section{Cell culture and transfection}

GIST-T1 cells were cultured in DMEM medium (Gibco, CA, USA) supplemented with $10 \%$ inactivated fetal bovine serum (FBS) (Gibco, CA, USA) in a humidified cell incubator with an atmosphere of $5 \% \mathrm{CO}_{2}$ at $37{ }^{\circ} \mathrm{C}$. GIST-T1 cells were transfected with TIPE2 overexpression plasmid or Rac1 siRNA using Lipofectamine 2000 according to the manufacturer's protocols (Invitrogen, Carlsbad, CA, USA). The mutant TIPE2 in which TIPE2 $\mathrm{N}$-terminal lysine or arginine residues, Lys-15, Lys-16, and Arg-24 were replaced with glutamine or alanine was generated by PCR-based site-directed mutagenesis as previously described [27]. The Rac1 siRNA sequences purchased from Sigma-Aldrich (St. Louis, USA) were as follows: 5'-GCAAACAGAUGUGUUCUUA-3', reverse $5^{\prime}$-UAAGAACACAUCUGUUUGC- ${ }^{\prime}$. Sequences for nonspecific negative control: forward $5^{\prime}$-UUCUCCGAA CGUGUCACGUTT- $3^{\prime}$, reverse 5'-ACGUGACACGUU CGGAGAATT-3'. NSC23766 (Calbiochem, San Diego, USA), a specific Rac1 inhibitor, was used to inhibit Rac1 activity in some experiments.

\section{RNA isolation and real-time quantitative PCR}

Total RNAs were extracted from transfected GIST-T1 cells using TRIzol reagent (Invitrogen, Carlsbad, CA, USA) and were reverse-transcribed into cDNA using a ReverTra Ace qPCR Kit (Toyobo, Osaka, Japan). Realtime PCR was performed using an UltraSYBR Mixture (CWBIO). The sequences of the sense and antisense primers were as follows: TIPE2: 5'-ACTGAGTAA GATGGCGGGTCG-3' ${ }^{\prime}$, and 5-TTCTGGCGAAAG CGGGTAG-3'; Rac1: 5'-ATGTCCGTGCAAAGTGGT ATC-3', and 5-CTCGGATCGCTTCGTCAAACA3'; GAPDH: 5'-AACGGATTTGGTCGTATTGGG-3', and $5^{\prime}$-CCTGGAAGATGGTGATGGGAT- ${ }^{\prime}$. Relative gene expression levels were normalized to GAPDH as control.

\section{Immunohistochemistry (IHC)}

IHC was performed using paraffin-embedded tissue sections. The sections were dewaxed and hydrated, followed by antigen retrieval (in $0.01 \mathrm{~mol} / \mathrm{L}$ citrate buffer solution, $\mathrm{pH}$ 6.0, heated to boiling for 2-3 $\mathrm{min}$ in a stainless steel pressure cooker). Endogenous peroxidase was blocked using a $3 \%$ hydrogen peroxide solution. The section was incubated with the blocking goat serum for $15 \mathrm{~min}$ and then immunostained with rabbit antibody against TIPE2 (dilution 1:100, Abcam, UK) or mouse antibody against Rac1 (dilution 1:100, Abcam, UK) at $4{ }^{\circ} \mathrm{C}$ overnight. Secondary staining was performed with HRP-conjugated anti-rabbit or anti-mouse IgG using a MaxVision Kit and a 3,5-diaminobenzidine (DAB) peroxidase substrate kit (Maixin Co, Fuzhou, China). The sections were then counterstained with hematoxylin.

\section{Evaluation of immunohistochemical staining}

Immunohistochemical staining was independently evaluated by two experienced pathologists in a blinded manner. Staining was semi-quantitatively scored based on both the staining intensity ( 0 , negative; 1 , very weak; 2 , weak; 3 , moderate; 4 , strong) and the percentage of positively stained cells $(0,0 \% ; 1,1-25 \% ; 2,26-50 \% ; 3$, 51-75\%; 4, 76-100\%). Both scores for each specimen were then combined to obtain the final score (IHC sum scores) of TIPE2 expression. The cut-off point for the sum of the scores was defined as follows: $0-2$, low expression; $3-8$, high expression.

\section{Cell viability assay}

A total number of 3000 GIST-T1 cells were seeded in 96-well plates in triplicate wells and cultured for the indicated times. Cell viability was evaluated using the Cell Counting Kit-8 (CCK8) (Beyotime, Haimen, China) assay 
according to the manufacturer's instructions. The absorbance was determined at $450 \mathrm{~nm}$.

\section{Colony formation assay}

GIST-T1 cells were seeded in six-well plates at a density of 500 cells per well and every 3 days the medium was replaced. Two weeks later, cells were washed by PBS and fixed with methanol for $10 \mathrm{~min}$, then stained with $1 \%$ crystal violet. Colonies that consisted of more than 50 cells were counted and calculated as a percentage of that to the control group.

\section{Transwell assays for cell migration and invasion}

The migration and invasion capacities of GIST-T1 cells were analyzed in 24-well Boyden chambers with $8-\mu \mathrm{m}$ pore size polycarbonate membranes (Costar, Acton, USA). For invasion assay, the membranes were precoated with $50 \mu \mathrm{g}$ Matrigel (BD Biosciences, San Diego, USA) to simulate matrix barriers. Cells $\left(5 \times 10^{5} / \mathrm{mL}\right)$ were resuspended in $200 \mu \mathrm{L}$ serum-free medium and placed in the upper chamber. The lower compartments were filled with $600 \mu \mathrm{L}$ medium with $10 \%$ FBS. The cells left on the upper surface of the membrane were removed after incubation. While the cells on the lower surface were fixed with methanol for $10 \mathrm{~min}$ and then stained with crystal violet for $20 \mathrm{~min}$. Stained cell counting was performed under a light microscope at $200 \times$ magnification.

\section{Caspase activity assay}

Caspase activity assays were carried out using a caspase fluorescent assay kit according to the manufacturer's instructions. In brief, GIST-T1 cells were homogenized on ice with lysis buffer. An aliquot of $50 \mu \mathrm{L}$ of supernatants was incubated with an equal volume of the reaction buffer containing fluorescent substrate at $37{ }^{\circ} \mathrm{C}$ for 1 to $2 \mathrm{~h}$. Enzymatic release of free fluorescent moiety was measured by a fluorometer.

\section{UPA and MMPs activity assays}

A total of $2 \times 10^{5}$ GIST-T1 cells were cultured in a sixwell culture plate and then treated with mock plasmid, TIPE2 overexpression plasmid, mutant plasmid, siNC or siRac1 for $24 \mathrm{~h}$. The levels of secreted uPA, MMP-2 and MMP-9 in the culture supernatant were determined using an enzyme-linked immunosorbent assay (ELISA) following the manufacturer's ELISA kit guidelines (R\&D).

\section{Statistical analysis}

A Chi square test of cross-tabulations and Fisher's exact test were used to determine the associations between TIPE2 expression and clinicopathological parameters. Quantitative data are presented as the mean \pm SD. The statistical significance was determined by two-tailed paired Student's t-test between two groups, and paired comparison between multiple groups or for different time points was conducted. Receiver operating characteristic (ROC) curve analysis was performed to assess the diagnostic value of TIPE2 in GIST. All statistical analysis was performed using SPSS 18.0 software (SPSS Inc., Chicago, USA), and a $P$ value $<0.05$ was considered statistically significant.

\section{Results}

The expression of TIPE2 was gradually decreased in accordance with GIST risk grades

IHC analysis was conducted using a total of 96 paraffinembedded GIST samples with different risk categories. As shown in Fig. 1, TIPE2 was predominantly expressed in the cytoplasm of the tumor cells. Strong TIPE2 staining was observed in very low risk GIST tissues (Fig. 1ac), while moderate TIPE2 expression was observed in low risk GIST tissues (Fig. 1d-f). TIPE2 expression was gradually decreased in accordance with GIST risk grades, as low and negative TIPE2 staining were shown in intermediate risk GIST tissues and high risk GIST tissues, respectively (Fig. 1g-i).

As shown in Table 1, although no significant association was shown between TIPE2 expression and Age $(P=0.2732)$, Gender $(P=0.8331)$ and Primary tumor site $(P=0.6585)$, TIPE2 expression was significantly associated with Tumor size $(P=0.0047)$, Mitotic count $(P=0.0005)$ and Risk category $(P=0.0006)$.

\section{TIPE2 was a promising biomarker for evaluating the risk grade of GIST}

As shown in Fig. 2a, statistical analysis of IHC sum scores among groups confirmed that TIPE2 expression was significantly correlated with the risk category of GIST. Moreover, the ratio of Ki-67 positive cells in low TIPE2 expression GIST tissues was higher compared with that of high TIPE2 expression GIST tissues (Fig. 2b). As shown in Fig. 2c, TIPE2 expression was lower in high risk GIST group. To further determine the diagnostic value of TIPE2 expression in predicting the risk grade of GIST, receiver operator characteristic (ROC) curves were constructed and the area under the curve (AUC) was calculated. The ROC curves suggested that the AUC value for predicting the risk grade of GIST was 0.754 (Fig. 2d, CI (95\%) 0.655-0.836, $P<0.0001$ ), with an estimated sensitivity and specificity of $74.67 \%$ and $71.43 \%$ respectively (Table 2).

\section{TIPE2 suppressed the proliferation, colony formation, migration and invasion of GIST-T1 cells}

Then the effect of TIPE2 on the malignant behaviors of GIST cells was explored in vitro. It was found that TIPE2 


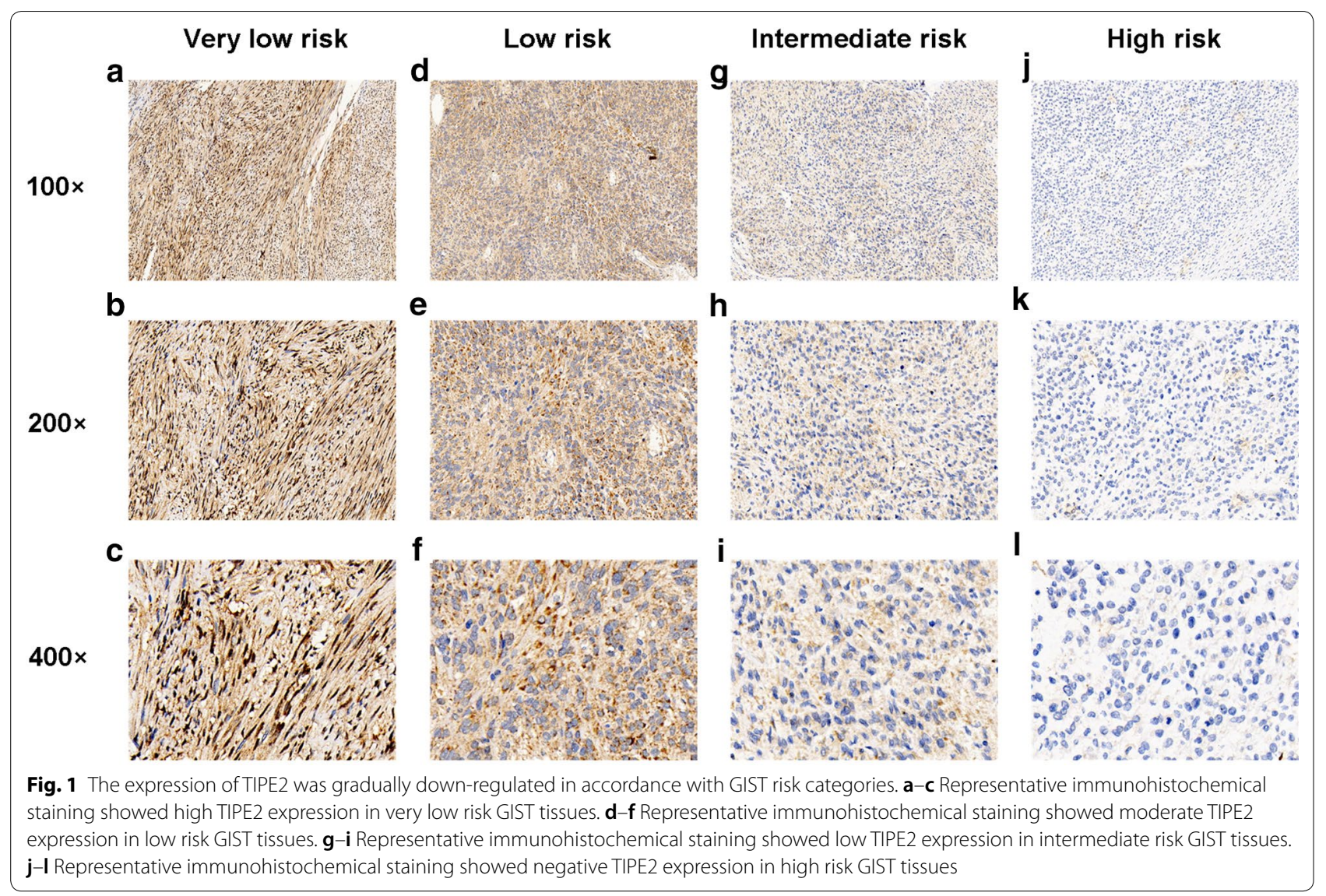

expression was low in GIST-T1 cells, so the TIPE2 overexpression plasmid was used. As shown in Fig. 3a, TIPE2 expression was up-regulated after transfection. CCK8 assays were used to evaluate the effects of TIPE2 on the viability of GIST cells, which revealed that TIPE2 overexpression suppressed GIST cell viability (Fig. 3b). Then colony formation assays demonstrated that TIPE2 also suppressed the colony formation capacity of GIST cells (Fig. 3c). Moreover, we performed Transwell migration and invasion assays in GIST-T1 cells after TIPE2 overexpression. As TIPE2 overexpression had no significant effect on cell viability within $24 \mathrm{~h}$, which eliminated the potential confounding influence of TIPE2 induced suppression of cell proliferation on cell migration and invasion. Then results showed that TIPE2 overexpression could suppress both the migration and invasion capacities of GIST cells (Fig. 3d, e).

\section{Rac1 was involved in the viability and invasiveness of GIST cells}

In order to investigate whether TIPE2 functions through targeting Rac1 in GIST, first we investigated the role of Rac1 in GIST. The expression of Rac1 in GIST tissues was detected using IHC. Results showed that Rac1 expression was gradually up-regulated in accordance with GIST risk grades, which was negatively associated with that of TIPE2 expression (Fig. 3f). Then we investigated the effect of Rac1 on the viability and invasiveness of GIST cells using Rac1 siRNA and NSC23766, a specific Rac1 activity inhibitor. CCK8 assays demonstrated that both Rac1 silencing and Rac1 inhibitor could suppress the viability of GIST cells (Fig. 3g, h). Further Transwell invasion assays found that Rac1 was also involved in the invasiveness of GIST cells (Fig. 3i, j).

\section{TIPE2 suppressed the viability and invasiveness of GIST cells via targeting Rac1}

To demonstrate whether TIPE2 functions through targeting Rac1 or not, the following experiments were performed. First, CCK8 assays were performed in GIST-T1 cells after co-transfected with TIPE2 overexpression plasmid and Rac1 siRNA. Results showed that Rac1 siRNA eliminated the inhibitory effect of TIPE2 on the viability of GIST-T1 cells (Fig. 4a). Moreover, GIST cells pretreated with NSC23766 also eliminated the effect of TIPE2 on cell viability (Fig. 4b). Further, the mutation of TIPE2 in sites which binds to Rac1, reversed the inhibitory effect of TIPE2 on cell viability (Fig. 4c). Similarly, 


\begin{tabular}{|c|c|c|c|c|}
\hline \multirow[t]{2}{*}{ Variable } & \multirow[t]{2}{*}{ Number } & \multicolumn{2}{|c|}{ TIPE2 expression } & \multirow[t]{2}{*}{$P$ value } \\
\hline & & Low & High & \\
\hline Number of patients & 96 & 34 & 62 & \\
\hline \multicolumn{5}{|l|}{ Age (years) } \\
\hline$<60$ & 59 & 18 & 41 & \multirow[t]{2}{*}{0.2732} \\
\hline$\geq 60$ & 37 & 16 & 21 & \\
\hline \multicolumn{5}{|l|}{ Gender } \\
\hline Male & 49 & 18 & 31 & \multirow[t]{2}{*}{0.8331} \\
\hline Female & 47 & 16 & 31 & \\
\hline \multicolumn{5}{|l|}{ Tumor size (cm) } \\
\hline$\leq 2$ & 20 & 3 & 17 & \multirow[t]{3}{*}{0.0047} \\
\hline $2-5$ & 42 & 12 & 30 & \\
\hline$>5$ & 34 & 19 & 15 & \\
\hline \multicolumn{5}{|c|}{ Mitotic count/50 HPFs } \\
\hline$\leq 5$ & 82 & 23 & 59 & \multirow[t]{2}{*}{0.0005} \\
\hline$>5$ & 14 & 11 & 3 & \\
\hline \multicolumn{5}{|l|}{ Primary tumor site } \\
\hline Gastric & 61 & 23 & 38 & \multirow[t]{2}{*}{0.6585} \\
\hline Non-gastric & 35 & 11 & 24 & \\
\hline \multicolumn{5}{|l|}{ Risk category } \\
\hline Very low risk & 22 & 3 & 19 & \multirow[t]{4}{*}{0.0006} \\
\hline Low risk & 35 & 10 & 25 & \\
\hline Intermediate risk & 18 & 6 & 12 & \\
\hline High risk & 21 & 15 & 6 & \\
\hline
\end{tabular}

Italic values represent significant value

both Rac1 siRNA and NSC23766 could eliminate the inhibitory effect of TIPE2 on the invasiveness of GIST-T1 cells, and mutant TIPE2 plasmid also reversed the effect of TIPE2 on cell invasion (Fig. 4d-f).

\section{TIPE2 induced apoptosis and decreased MMP-9 expression of GIST cells in Rac1 dependent manner}

Previous research demonstrated that Rac1 is important in regulating apoptosis $[25,26]$. To investigate whether TIPE2 suppressed the viability of GIST cells via inducing apoptosis, caspase- 9 and caspase- 3 activities of GIST-T1 cells were detected after TIPE2 overexpression. Results showed that TIPE2 increased caspase- 9 and caspase-3 activity in GIST cells (Fig. 5a). To further explore the role of Rac1 in TIPE2 induced apoptosis, Rac1 siRNA and inhibitor were used. It was found that both Rac1 silencing and NSC23766 could increase the activities of caspase-9 and caspase-3 (Fig. 5b, c). More importantly, mutant TIPE2 reversed the effect of TIPE2 on apoptosis (Fig. 5d), indicating that TIPE2 induced apoptosis of GIST cells via inhibiting Rac1.

It is evident that Rac1 plays a pivotal role in regulating tumor invasiveness, and uPA and MMPs have been demonstrated to be the downstream effectors of Rac1 signaling $[28,29]$. Then the effect of TIPE2 on the expression of uPA, MMP-2 and MMP-9 was detected. It was found that TIPE2 overexpression decreased the expression of MMP-9 in GIST cells, while no significant changes were shown on uPA and MMP-2 expression (Fig. 5e). Moreover, both Rac1 siRNA and Rac1 inhibitor also suppressed MMP-9 expression (Fig. 5f, g). Furthermore, the mutation of TIPE2 in sites which binds to Rac1 reversed the inhibitory effect of TIPE2 on MMP-9 expression (Fig. 5h). In addition, the expression of MMP-9 in GIST tissues was negatively associated with TIPE2 expression (Fig. 5i, j).

\section{Discussion}

The notion of GISTs was preliminarily introduced in the 1980 s, but these neoplasms were not widely recognized until the start of the 21st century, when the molecular biology and genetic diagnosis were generalized [30]. Nowadays, the National Institutes of Health (NIH) consensus criteria, Armed Forces Institute of Pathology (AFIP) criteria, and modified NIH criteria are frequently used to estimate the risk category of GISTs $[4,8,31]$. Although the prognostic value of these schemes has been validated, more confounding factors that may improve the evaluation of GIST risk grade are still needed [32, 33].

TIPE2 belongs to the TIPE family and was preliminarily identified as an immune negative regulator. Recently, TIPE2 has been found to be involved in various of epithelium derived carcinomas [18, 19, 29, 34]. However, the expression and roles of TIPE2 in GIST, a special kind of mesenchyme originated neoplasm, are largely unknown. Here we found that TIPE2 was highly expressed in the very low risk GIST tissues, and TIPE2 expression was gradually decreased in accordance with GIST risk grades. Nowadays, the most important independent prognostic factor for evaluating GIST risk grade is a high tumor mitotic rate [32, 35]. TIPE2 expression was negatively associated with tumor size and mitotic count, as well as the ratio of Ki-67 positive cells, which has been suggested as an alternative for mitosis counting [36]. Moreover, further analysis revealed that TIPE2 may serve as a promising biomarker for evaluating the risk grade of GIST.

Based on our IHC findings, we also extended the observations to investigate the effect of TIPE2 on the malignant behaviors of GIST cells. Likewise, TIPE2 acted as a tumor suppressor in GIST as well, for it suppressed the proliferation, colony formation, migration and invasion of GIST cells. Of note, TIPE2 effectively inhibited the invasiveness of GIST cells. Enhanced invasiveness usually leads to tumor metastasis, which is the main reason for the poor prognosis of GIST patients [32, 37]. Therefore, 

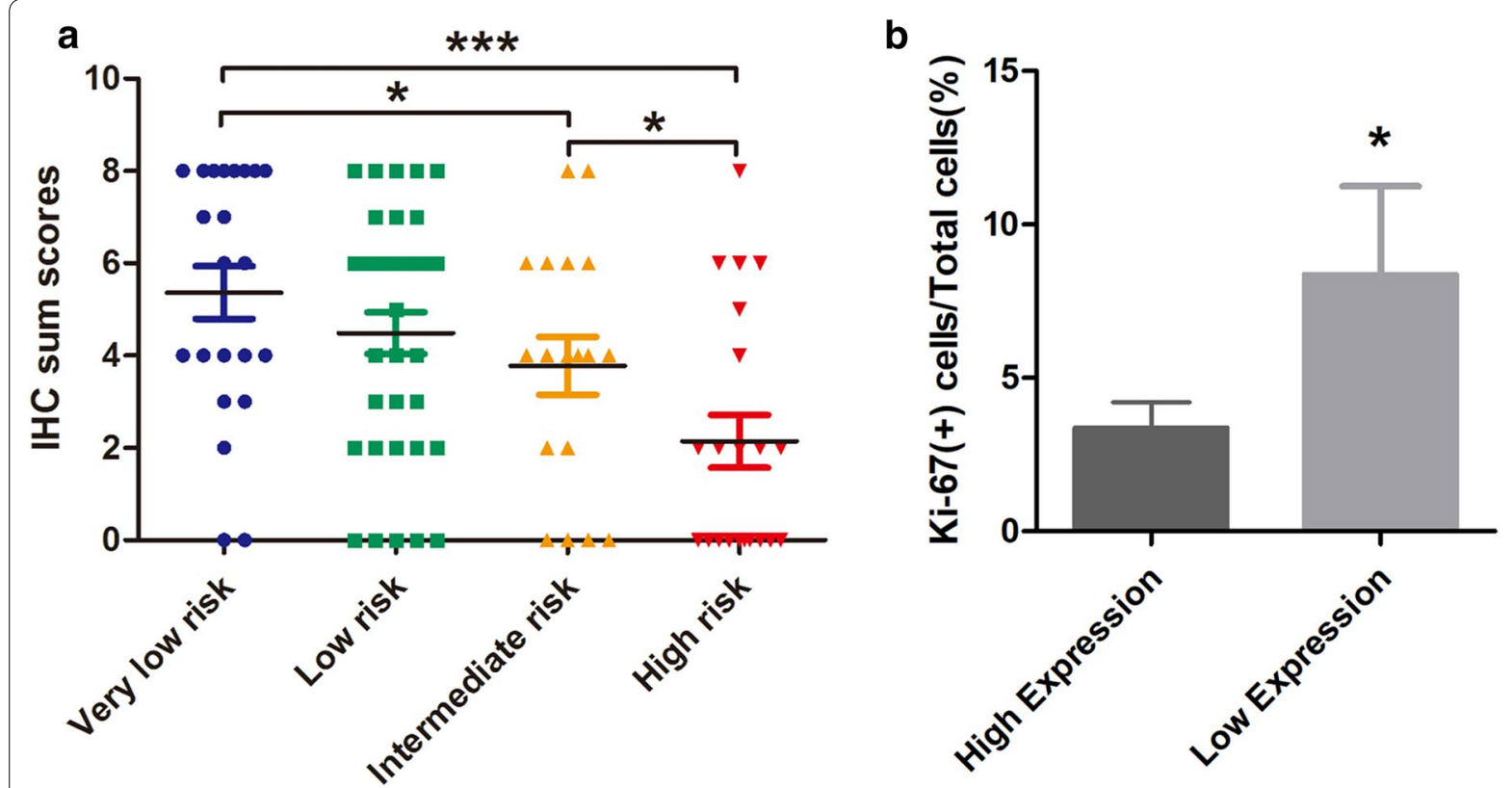

C

d
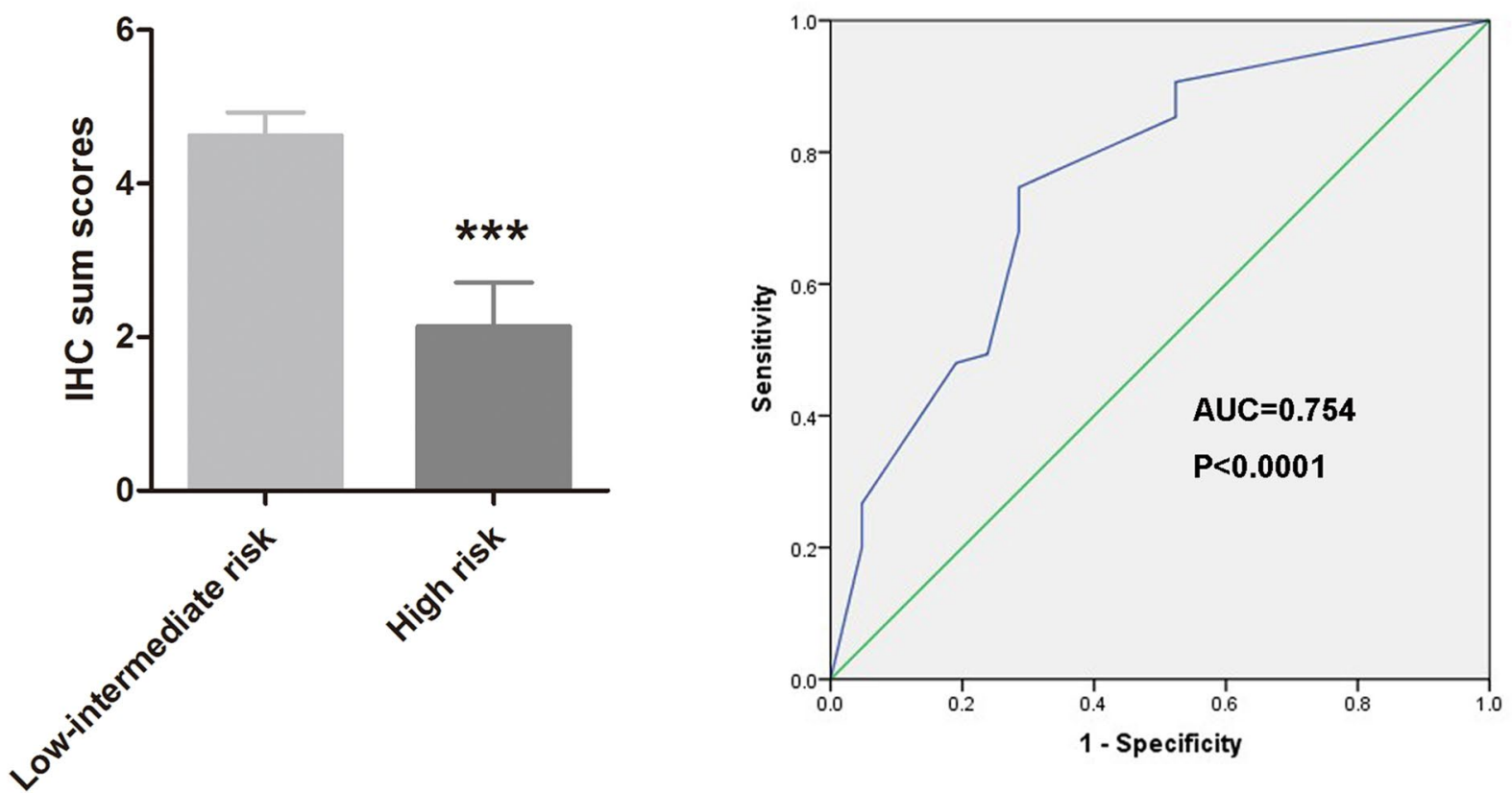

Fig. 2 Statistical results of TIPE2 expression in GIST tissues and ROC curves curve to assess the diagnostic value of TIPE2 in GIST. a IHC sum sCores showed that TIPE2 expression was gradually down-regulated in accordance with GIST risk grades. $\mathbf{b} I \mathrm{HC}$ sum scores were used to assess the association between TIPE2 expression and the ratio of Ki-67 positive cells. $\mathbf{c}$ IHC sum scores showed a significant decrease of TIPE2 expression in high risk GIST tissues. $\mathbf{d}$ The ROC curves showed strong separation between low-intermediate risk GIST tissues and high risk GIST tissues, with an AUC of $0.754(P<0.001)$. ${ }^{*} P<0.05 ; * * * P<0.001$

TIPE2 might represent a potential target for the treatment of GIST, and explicating the detailed mechanisms is indispensable.
Previous research found that TIPE2 functions through binding with Rac1 and subsequently inhibiting its activity [15, 17, 27, 38]. It has been reported that Rac1 signaling plays a pivotal role in the invasion 
Table 2 Sensitivity, specificity, and positive and negative predictive values for evaluating GISTs risk category using TIPE2 IHC expression

\begin{tabular}{llllllllll}
\hline IHC scores & Sensitivity & $\mathbf{9 5 \%} \mathbf{C l}$ & Specificity & $\mathbf{9 5 \%} \mathrm{Cl}$ & $\mathbf{P P V}$ & $\mathbf{9 5 \%} \mathrm{Cl}$ & $\mathbf{N P V}$ & $\mathbf{9 5 \%} \mathrm{Cl}$ & Significance \\
\hline$\leq 2$ & 74.67 & $63.3-84.0$ & 71.43 & $47.8-88.7$ & 90.3 & $80.1-96.4$ & 44.1 & $27.2-62.1$ & High risk prediction \\
\hline
\end{tabular}

PPV positive predictive value, NPV negative predictive value

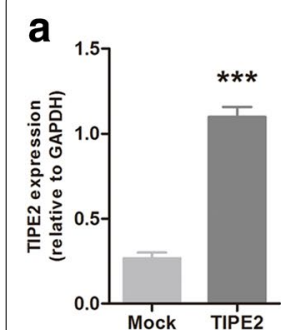

b

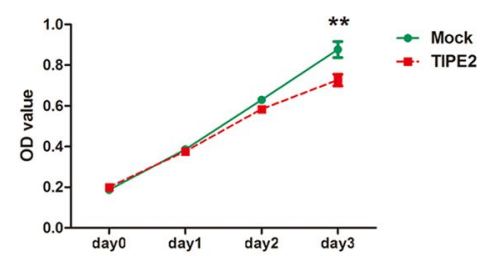

d
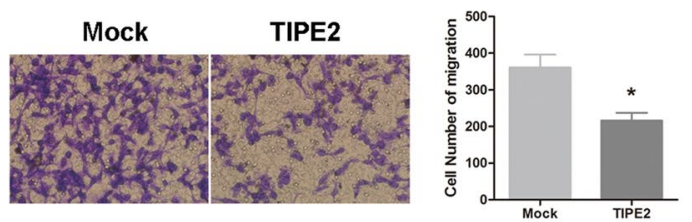

f

Very low risk
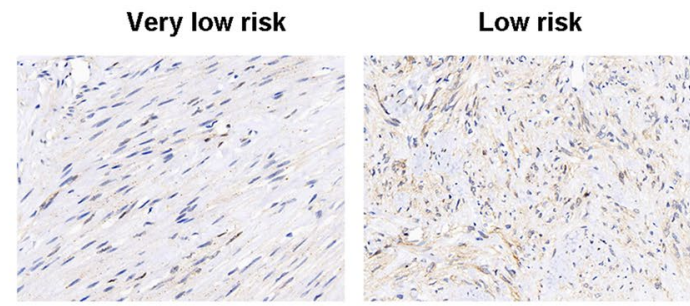

C
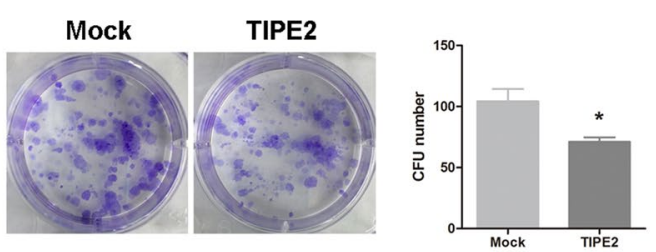

e
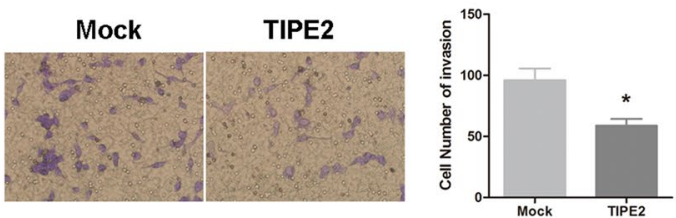

Intermediate risk

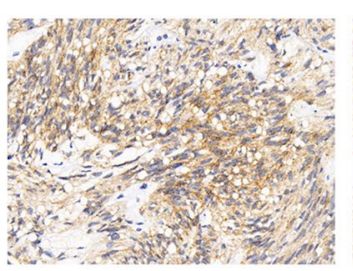

High risk

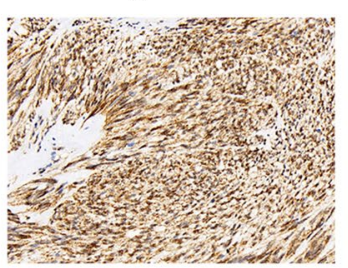

g

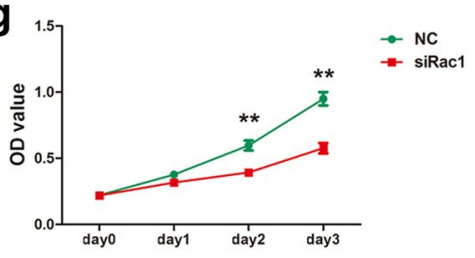

h

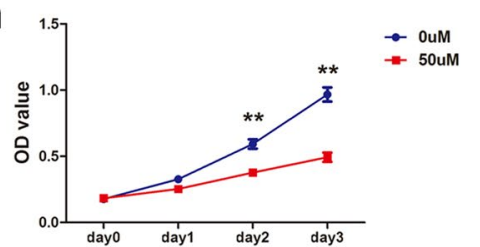

i

NC

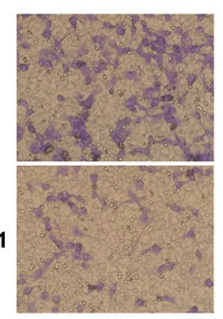

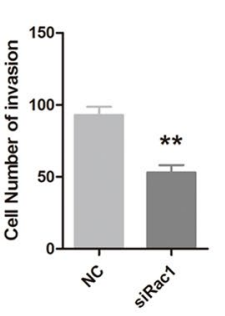

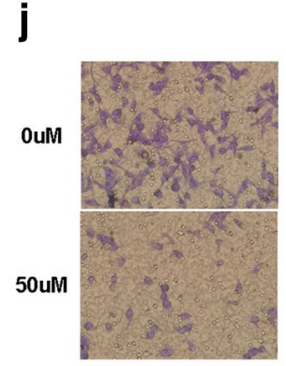

Fig. 3 TIPE2 overexpression could suppress the proliferation, colony formation, migration and invasion of GIST-T1 cells by targeting Rac1. a Real-time PCR showed that TIPE2 expression was low in GIST-T1 cells and TIPE2 expression was up-regulated after transfection of TIPE2 overexpression plasmid. b CCK8 assays revealed that TIPE2 overexpression could suppress the viability of GIST-T1 cells. c After TIPE2 overexpression, colony formation assays were conducted to evaluated the colony formation capacity of GIST-T1 cells. d, e GIST-T1 cells transfected with the TIPE2 overexpression plasmid were used for Transwell migration and invasion assays. f Representative immunohistochemical staining showed that Rac1 expression was gradually up-regulated in accordance with GIST risk grades. $\mathbf{g}$ CCK8 assay was conducted to evaluate the viability of GIST-T1 cells after transfection with Rac1 siRNA. h CCK8 assay was conducted to evaluate the viability of GIST-T1 cells after treatment of NSC23766, a specific Rac1 inhibitor. i Transwell invasion assay revealed that Rac1 silencing decreased the invasiveness of GIST cells. $\mathbf{j}$ Transwell invasion assay was conducted to evaluate the invasiveness of GIST cells after treatment of NSC23766. ${ }^{* *} P<0.01$. Data represent the mean \pm SD of three independent experiments. ${ }^{*} P<0.05 ;{ }^{* *} P<0.01 ;{ }^{* * *} P<0.001$ 

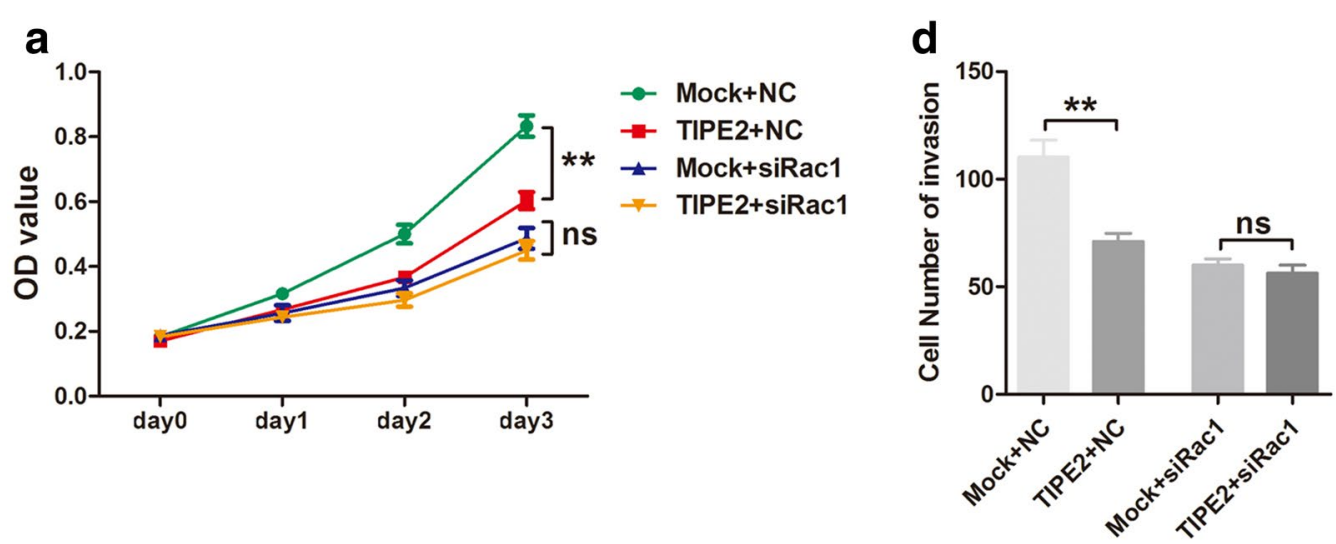

b

e
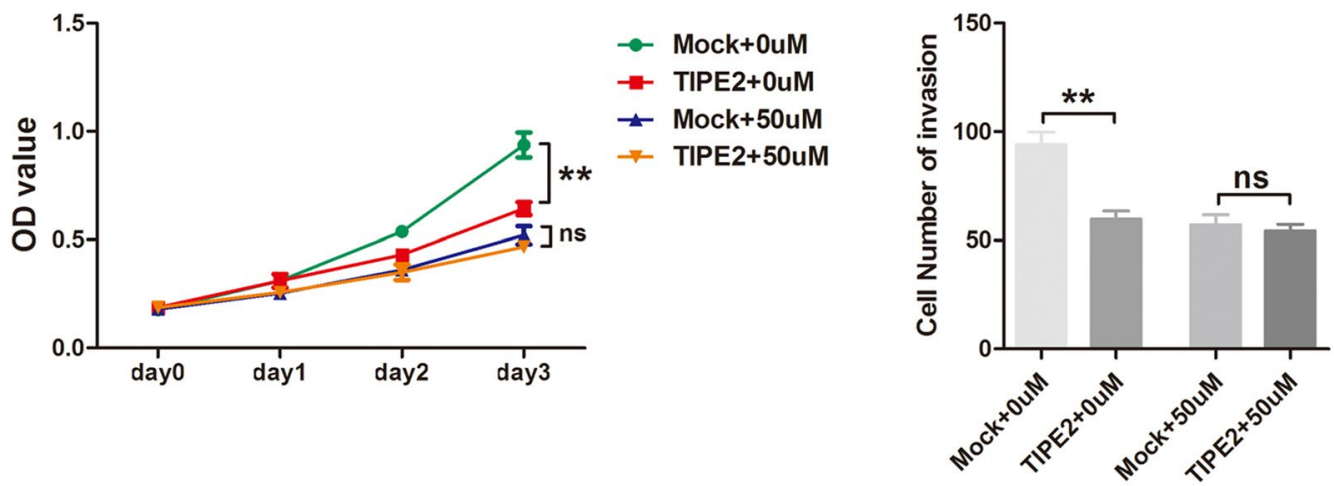

C

f
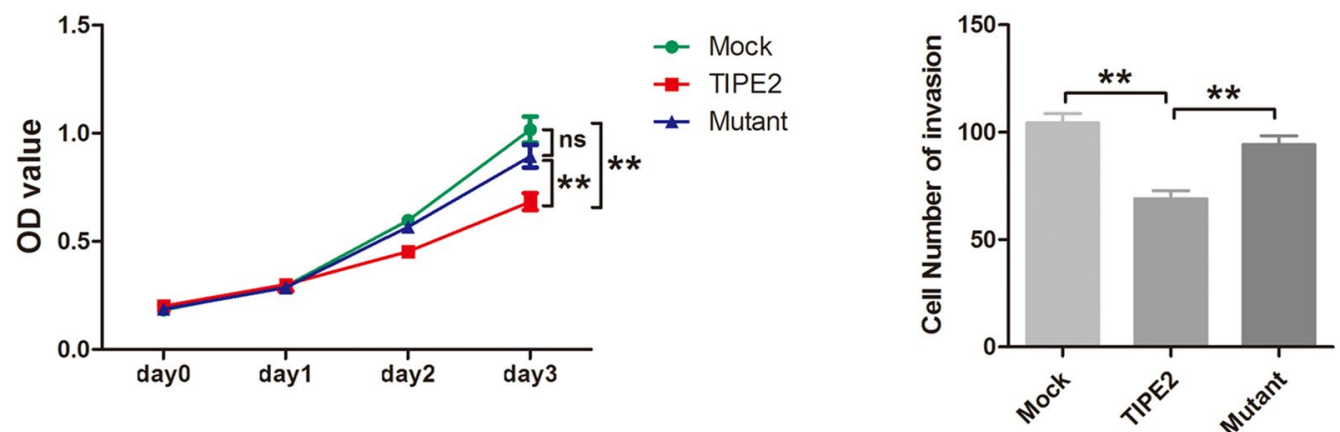

Fig. 4 TIPE2 suppressed the proliferation and invasion of GIST cells by inhibiting Rac1. a After co-transfected with Rac1-specific siRNA and TIPE2 overexpression plasmid, GIST-T1 cells were applied to CCK8 assays. b After treatment with NSC23766, GIST-T1 cells were transfected with the TIPE2-overexpressing plasmid, and cell viability was measured by CCK8 assays. c GIST-T1 cells that transfected with mock, wild type TIPE2 and mutant TIPE2 plasmids were used for CCK8 assays. d GIST-T1 cells that co-transfected with Rac1-specific siRNA and TIPE2 overexpression plasmid were applied to Transwell invasion assays. e After treatment with NSC23766, GIST-T1 cells were transfected with the TIPE2-overexpressing plasmid, and cell invasiveness was measured by Transwell invasion assays. F. GIST-T1 cells that transfected with mock, wild type TIPE2 and mutant TIPE2 plasmids were used for Transwell invasion assays. ${ }^{* *} P<0.01$

and metastasis of GIST [39]. Nevertheless, the detailed roles of Rac1 in GIST are unclear. In the present study, the expression and roles of Rac1 in GIST was examined. It was found that Rac1 expression was gradually increased in accordance with GIST risk grades and negatively associated with that of TIPE2. Our further experiments demonstrated that Rac1 was also involved in the proliferation and invasiveness of GIST cells. Moreover, TIPE2 suppressed the proliferation and invasion of GIST cells via targeting Rac1.

TIPE2 has been demonstrated to promote the apoptosis by regulating caspase- 9 and caspase- 3 in both lung 

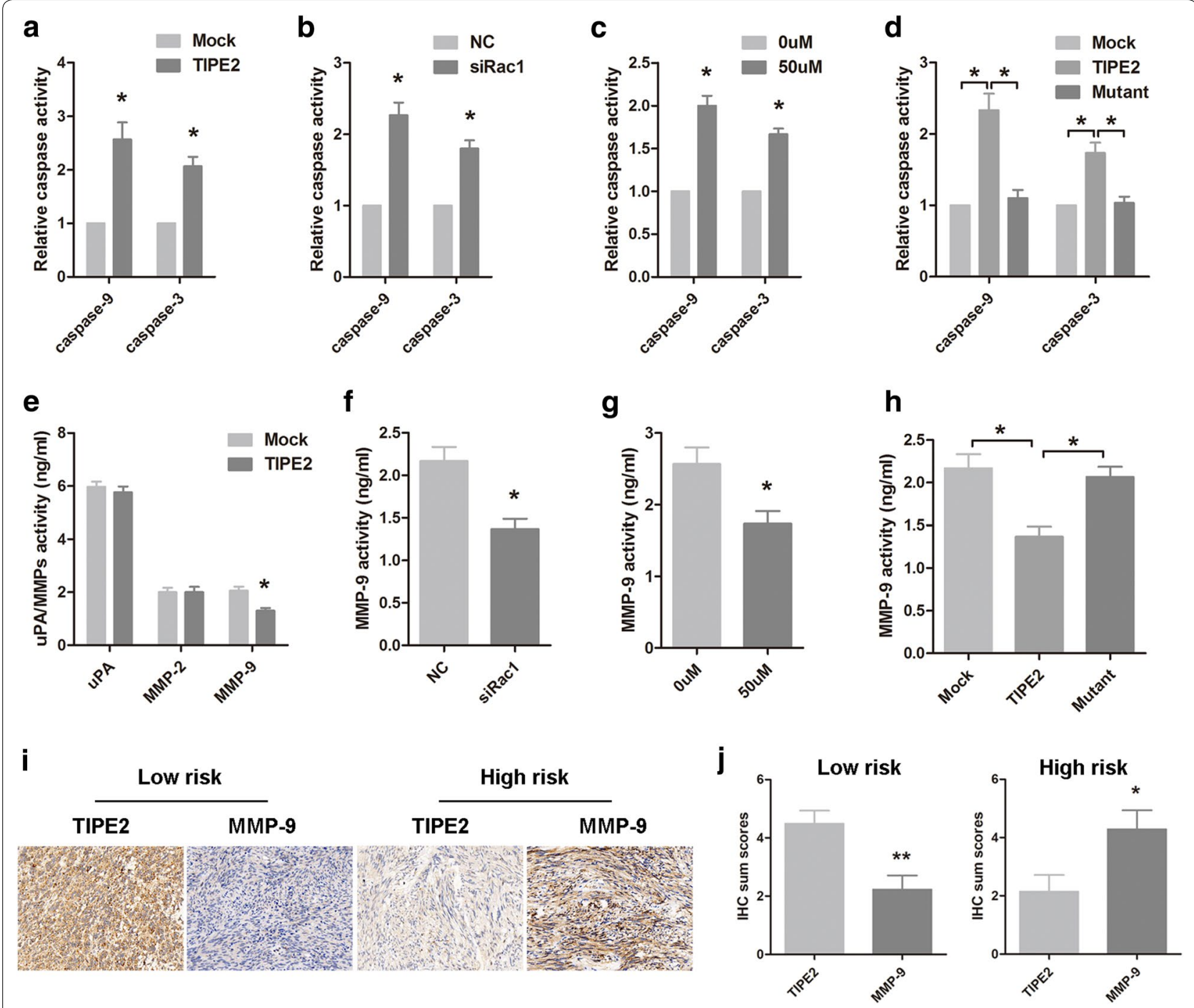

Fig. 5 TIPE2 induced apoptosis and suppressed the expression of MMP9 in a Rac1-dependent manner. a Caspase-9/3 activity of GIST-T1 cells that transfected with TIPE2 overexpression plasmid were examined using the fluorescent caspase activity assay. $\mathbf{b}$ Caspase-9/3 activity of GIST-T1 cells that transfected with Rac1 siRNA were examined using the fluorescent caspase activity assay. c Caspase-9/3 activity of GIST-T1 cells that pretreated with NSC2366 were examined using the fluorescent caspase activity assay. $\mathbf{d}$ GIST-T1 cells that transfected with Mock, TIPE2 overexpression plasmid and TIPE2 mutant plasmid were applied to the fluorescent caspase activity assays. e The protein level of uPA, MMP-2 and MMP-9 were detected by ELISA in GIST-T1 cells transfected with TIPE2 overexpression plasmid. $\mathbf{f}$ The protein level of MMP-9 was detected by ELISA in GIST-T1 cells transfected with Rac1 siRNA. G. The protein level of MMP-9 was examined by ELISA in GIST-T1 cells pretreated with NSC23766. H. GIST-T1 cells that transfected with Mock, TIPE2 overexpression plasmid and TIPE2 mutant plasmid were used for MMP-9 activity assays. $* P<0.05$. i Representative immunohistochemical staining showed high TIPE2 expression and low MMP-9 expression in low risk GIST tissues, while the MMP-9 expression was high in high risk GIST tissues. $\mathbf{j}$ IHC sum scores showed low MMP-9 expression and high TIPE2 expression in low risk GIST tissues, while high MMP-9 expression and low TIPE2 expression in high risk GIST tissues

cancer and gastric cancer [40, 41]. TIPE2 also decreased the expression of uPA and MMP-9 in HCC via inhibiting the activity of Rac1 [15]. Here our results showed that TIPE2 induced the expression of caspase-9, caspase-3 and decreased MMP-9 expression in GIST cells. Several classical molecules that contributing to apoptosis and cell invasiveness have been demonstrated to be downstream effectors of Rac1 [20, 28]. Consistent with the aforementioned results, our data demonstrated that TIPE2 induced apoptosis and decreased MMP-9 expression of GIST cells in Rac1 dependent manner.

One of the greatest challenge for the treatment of GISTs in clinics is chemoresistance [1], the role of TIPE2 in the chemoresistance and its related 
mechanisms (such as its effect on cancer stem-like cells etc.) warrant further study. Moreover, the relationship between TIPE2 expression and the prognosis of GIST patients still needs to be further investigated. In addition, as the epitope for generating the antibody of TIPE2 was the same for both wild type TIPE2 and mutation TIPE2, it is difficult to detect the expression of mutant TIPE2 in GIST tissues.

In conclusion, the present study demonstrated that TIPE2 serve as a potential biomarker for evaluating the risk grade of GIST, which enriched the risk criteria for GIST. Moreover, by regulating tumor proliferation and invasiveness, forced TIPE2 expression may be considered as a potential therapeutic strategy in controlling the progression of GIST.

\section{Abbreviations}

GISTs: gastrointestinal stromal tumors; TIPE2: tumor necrosis factor-alphainduced protein 8-like 2; ICC: interstitial cells of Cajal; PDGFRA: platelet-derived growth factor receptor alpha; HCC: hepatocellular carcinoma; NSCLC: nonsmall cell lung cancer; MMPs: matrix metalloproteinase; UPA: urokinase plasminogen activator; FBS: fetal bovine serum; IHC: immunohistochemistry; ROC: receiver operating characteristic; AUC: area under the curve; $\mathrm{NIH}$ : National Institutes of Health; AFIP: Armed Forces Institute of Pathology.

\section{Authors' contributions}

$Y Z$ conceived and designed the research; ZL and WZ performed the experiments; YL, SC and SL contributed to make the table and figures; $L N, X J$ and $Z \mathrm{~L}$ contributed to the statistical analysis; $X X$ and $Y L$ contributed to the IHC analysis; $Z \mathrm{~L}$ wrote the paper; $Y Z$ revised the paper. All authors read and approved the final manuscript.

\section{Author details}

${ }^{1}$ Department of General Surgery, Affiliated Hospital of Qingdao University, 16\# Jiangsu Road, Qingdao, Shandong, People's Republic of China. ${ }^{2}$ Department of Emergency Medicine, Affiliated Hospital of Qingdao University, Qingdao, China. ${ }^{3}$ Department of Emergency General Surgery, Affiliated Hospital of Qingdao University, Qingdao, China. ${ }^{4}$ Department of Oncology, Affiliated Hospital of Qingdao University, Qingdao, China. ${ }^{5}$ Department of Pathology, Affiliated Hospital of Qingdao University, Qingdao, China.

\section{Competing interests}

The authors declare that they have no competing interests.

\section{Availability of data and materials}

Please contact the corresponding author for data on reasonable request.

\section{Consent for publication}

Not applicable.

\section{Ethics approval and consent to participate}

The study was approved by the Institutional Review Board of Shandong University, China. Written informed consent was obtained from all subjects.

\section{Funding}

This work was supported by the National Natural Science Foundation of China (Nos. 81270449, 81572314), Shandong Provincial Natural Science Foundation, China (No. ZR2012HM046), Key Research Project of Shandong Province, China (2016GGB01022), Qingdao Minsheng Science and Technology Foundation, Shandong, China (No. 14-2-3-5-nsh) and Qingdao Science and Technology Plan Project (No. 13-1-4-220-jch).

\section{Publisher's Note}

Springer Nature remains neutral with regard to jurisdictional claims in published maps and institutional affiliations.

Received: 10 September 2018 Accepted: 29 November 2018

Published online: 05 December 2018

\section{References}

1. Joensuu H, Hohenberger P, Corless CL. Gastrointestinal stromal tumour. Lancet. 2013;382:973-83.

2. Miettinen M, Lasota J. Histopathology of gastrointestinal stromal tumor. J Surg Oncol. 2011;104:865-73.

3. Corless CL, Barnett CM, Heinrich MC. Gastrointestinal stromal tumours: origin and molecular oncology. Nat Rev Cancer. 2011;11:865-78.

4. Joensuu H. Risk stratification of patients diagnosed with gastrointestinal stromal tumor. Hum Pathol. 2008;39:1411-9.

5. von Mehren M, Randall RL, Benjamin RS, Boles S, Bui MM, Conrad EU 3rd, et al. Soft tissue sarcoma, version 2.2016, NCCN clinical practice guidelines in oncology. J Natl Compr Cancer Netw. 2016;14:758-86.

6. Agaimy A, Wunsch PH. Lymph node metastasis in gastrointestinal stromal tumours (GIST) occurs preferentially in young patients $\leq 40$ years: an overview based on our case material and the literature. Langenbeck's Arch Surg. 2009;394:375-81.

7. Hohenberger P, Ronellenfitsch U, Oladeji O, Pink D, Strobel P, Wardelmann $E$, et al. Pattern of recurrence in patients with ruptured primary gastrointestinal stromal tumour. Br J Surg. 2010;97:1854-9.

8. Fletcher CD, Berman JJ, Corless C, Gorstein F, Lasota J, Longley BJ, et al. Diagnosis of gastrointestinal stromal tumors: a consensus approach. Hum Pathol. 2002;33:459-65.

9. Lou Y, Liu S. The TIPE (TNFAIP8) family in inflammation, immunity, and cancer. Mol Immunol. 2011;49:4-7.

10. Zhang X, Wang J, Fan C, Li H, Sun H, Gong S, et al. Crystal structure of TIPE2 provides insights into immune homeostasis. Nat Struct Mol Biol. 2009;16:89-90.

11. Sun H, Gong S, Carmody RJ, Hilliard A, Li L, Sun J, et al. TIPE2, a negative regulator of innate and adaptive immunity that maintains immune homeostasis. Cell. 2008;133:415-26.

12. Xi W, Hu Y, Liu Y, Zhang J, Wang L, Lou Y, et al. Roles of TIPE2 in hepatitis B virus-induced hepatic inflammation in humans and mice. Mol Immunol. 2011;48:1203-8.

13. Ma Y, Liu X, Wei Z, Wang $X$, Wang Z, Zhong W, et al. The expression and significance of TIPE2 in peripheral blood mononuclear cells from asthmatic children. Scand J Immunol. 2013;78:523-8.

14. Qian J, Meng Z, Guan J, Zhang Z, Wang Y. Expression and roles of TIPE2 in autoimmune hepatitis. Exp Ther Med. 2017;13:942-6.

15. Cao X, Zhang L, Shi Y, Sun Y, Dai S, Guo C, et al. Human tumor necrosis factor (TNF)-alpha-induced protein 8-like 2 suppresses hepatocellular carcinoma metastasis through inhibiting Rac1. Mol Cancer. 2013;12:149.

16. Lu Q, Liu Z, Li Z, Chen J, Liao Z, Wu WR, et al. TIPE2 overexpression suppresses the proliferation, migration, and invasion in prostate cancer cells by inhibiting PI3K/Akt signaling pathway. Oncol Res. 2016;24:305-13.

17. Li Z, Guo C, Liu X, Zhou C, Zhu F, Wang X, et al. TIPE2 suppresses angiogenesis and non-small cell lung cancer (NSCLC) invasiveness via inhibiting Rac1 activation and VEGF expression. Oncotarget. 2016;7:62224-39.

18. Goldsmith JR, Chen YH. Regulation of inflammation and tumorigenesis by the TIPE family of phospholipid transfer proteins. Cell Mol Immunol. 2017; 14:1026.

19. Zhao Q, Zhao M, Dong T, Zhou C, Peng Y, Zhou X, et al. Tumor necrosis factor-alpha-induced protein-8 like-2 (TIPE2) upregulates p27 to decrease gastic cancer cell proliferation. J Cell Biochem. 2015;116:1121-9.

20. Colley NJ. Cell biology. Actin' up with Rac1. Science. 2000;290:1902-3.

21. Worthylake DK, Rossman KL, Sondek J. Crystal structure of Rac1 in complex with the guanine nucleotide exchange region of Tiam1. Nature. 2000;408:682-8.

22. Navarro-Lerida I, Pellinen T, Sanchez SA, Guadamillas MC, Wang Y, Mirtti T, et al. Rac1 nucleocytoplasmic shuttling drives nuclear shape changes and tumor invasion. Dev Cell. 2015;32:318-34. 
23. Keely PJ, Westwick JK, Whitehead IP, Der CJ, Parise LV. Cdc42 and Rac1 induce integrin-mediated cell motility and invasiveness through $\mathrm{PI}(3) \mathrm{K}$. Nature. 1997;390:632-6.

24. Murthy S, Ryan A, He C, Mallampalli RK, Carter AB. Rac1-mediated mitochondrial $\mathrm{H} 2 \mathrm{O} 2$ generation regulates MMP-9 gene expression in macrophages via inhibition of SP-1 and AP-1. J Biol Chem. 2010;285:25062-73.

25. Zhang Z, Liang X, Gao L, Ma H, Liu X, Pan Y, et al. TIPE1 induces apoptosis by negatively regulating Rac1 activation in hepatocellular carcinoma cells. Oncogene. 2015;34:2566-74.

26. Bhat SS, Parray AA, Mushtaq U, Fazili KM, Khanday FA. Actin depolymerization mediated loss of SNTA1 phosphorylation and Rac1 activity has implications on ROS production, cell migration and apoptosis. Apoptosis. 2016:21:737-48.

27. Wang Z, Fayngerts S, Wang P, Sun H, Johnson DS, Ruan Q, et al. TIPE2 protein serves as a negative regulator of phagocytosis and oxidative burst during infection. Proc Natl Acad Sci USA. 2012;109:15413-8.

28. Gonzalez-Villasana V, Fuentes-Mattei E, Ivan C, Dalton HJ, RodriguezAguayo C, Fernandez-de Thomas RJ, et al. Rac1/Pak1/p38/MMP-2 axis regulates angiogenesis in ovarian cancer. Clin Cancer Res. 2015;21:2127-37.

29. Prakobwong S, Yongvanit P, Hiraku Y, Pairojkul C, Sithithaworn P, Pinlaor P, et al. Involvement of MMP-9 in peribiliary fibrosis and cholangiocarcinogenesis via Rac1-dependent DNA damage in a hamster model. Int $J$ Cancer. 2010;127:2576-87.

30. Mazur MT, Clark HB. Gastric stromal tumors: reappraisal of histogenesis. Am J Surg Pathol. 1983;7:507-19.

31. Miettinen M, Lasota J. Gastrointestinal stromal tumors: pathology and prognosis at different sites. Semin Diagn Pathol. 2006;23:70-83.

32. Joensuu H, Vehtari A, Riihimaki J, Nishida T, Steigen SE, Brabec P, et al. Risk of recurrence of gastrointestinal stromal tumour after surgery: an analysis of pooled population-based cohorts. Lancet Oncol. 2012;13:265-74.
33. Rutkowski P, Bylina E, Wozniak A, Nowecki ZI, Osuch C, Matlok M, et al. Validation of the Joensuu risk criteria for primary resectable gastrointestinal stromal - the impact of tumour rupture on patient outcomes. Eur J Surg Oncol. 2011;37:890-6.

34. Li XM, Su JR, Yan SP, Cheng ZL, Yang TT, Zhu Q. A novel inflammatory regulator TIPE2 inhibits TLR4-mediated development of colon cancer via caspase-8. Cancer Biomark. 2014;14:233-40.

35. Dematteo RP, Gold JS, Saran L, Gonen M, Liau KH, Maki RG, et al. Tumor mitotic rate, size, and location independently predict recurrence after resection of primary gastrointestinal stromal tumor (GIST). Cancer. 2008;112:608-15.

36. Liang YM, Li XH, Li WM, Lu YY. Prognostic significance of PTEN, Ki-67 and CD44s expression patterns in gastrointestinal stromal tumors. World J Gastroenterol. 2012;18:1664-71.

37. Woodall CE 3rd, Brock GN, Fan J, Byam JA, Scoggins CR, McMasters KM, et al. An evaluation of 2537 gastrointestinal stromal tumors for a proposed clinical staging system. Arch Surg. 2009;144:670-8.

38. Sun H, Zhuang G, Chai L, Wang Z, Johnson D, Ma Y, et al. TIPE2 controls innate immunity to RNA by targeting the phosphatidylinositol 3-kinaseRac pathway. J Immunol. 2012;189:2768-73.

39. Ma MZ, Zhuang C, Yang XM, Zhang ZZ, Ma H, Zhang WM, et al. CTHRC1 acts as a prognostic factor and promotes invasiveness of gastrointestinal stromal tumors by activating Wnt/PCP-Rho signaling. 2014;16:265-78, 278.e1-13.

40. Zhu Y, Tao M, Wu J, Meng Y, Xu C, Tian Y, et al. Adenovirus-directed expression of TIPE2 suppresses gastric cancer growth via induction of apoptosis and inhibition of AKT and ERK1/2 signaling. Cancer Gene Ther. 2016;23:98-106.

41. Liu QQ, Zhang FF, Wang F, Qiu JH, Luo CH, Zhu GY, et al. TIPE2 inhibits lung cancer growth attributing to promotion of apoptosis by regulating some apoptotic molecules expression. PLoS ONE. 2015;10:e0126176.
Ready to submit your research? Choose BMC and benefit from:

- fast, convenient online submission

- thorough peer review by experienced researchers in your field

- rapid publication on acceptance

- support for research data, including large and complex data types

- gold Open Access which fosters wider collaboration and increased citations

- maximum visibility for your research: over $100 \mathrm{M}$ website views per year

At $\mathrm{BMC}$, research is always in progress.

Learn more biomedcentral.com/submissions 